\title{
NOUVELLe
}

\section{Les CAR-T cells à NKG2D en immunothérapie contre le carcinome hépatocellulaire}

Gilles Roussine Codo ${ }^{1}$, Sarra Khennas ${ }^{2}$

\author{
${ }^{1}$ Master 2 Immunologie Intégrative et Systémique, \\ Mention BMC, Sorbonne Université, Paris, France. \\ ${ }^{2}$ Master 2 Immunologie Translationnelle et Biothérapies, \\ Mention BMC, Sorbonne Université, Paris, France. \\ gilles.codo@etu.upmc.fr \\ sarra.khennas@gmail.com
}

Cellules CAR-T dans les cancers : quelles avancées?

Les CAR-T cells, pour chimeric antigen receptor $T$ cells, sont des lymphocytes $T$ (LT) autologues portant un récepteur construit par ingénierie génétique, spécifique d'un antigène tumoral. Le CAR est une protéine de fusion comportant 1) un domaine extracellulaire de fixation à l'antigène $(\mathrm{Ag})$ composé d'un fragment d'anticorps appelé scFv (pour single chain fragment variable), contenant un domaine VH et un domaine VL associés par un polypeptide ; 2) un domaine intracellulaire composé d'un ou de deux domaines de co-stimulation activatrice CD28, 0X40/ CD134 ou 4-1BB/CD137, associés à un domaine de signalisation, la chaîne $\zeta$ du CD3 [1]. Les CAR-T cells peuvent reconnaître l'antigène indépendamment d'un processus de présentation de peptides par les molécules du CMH (complexe majeur d'histocompatibilité), ce qui est avantageux pour les défenses contre les tumeurs, qui peuvent exprimer des niveaux faibles des molécules du CMH [1].

Depuis leur génération en 1989 [2] $(\rightarrow)$ les CAR-T cells ont fait $(\rightarrow)$ Voir la synthèse leurs preuves, parti- de V. Catros, $\mathrm{m} / \mathrm{s}$ culièrement dans les $n^{\circ} 4$, avril 2019, hémopathies malignes page 316 avec l'approbation en 2017 par la FDA (food and drug administration) de la commercialisation du tisagenlecleucel pour le traitement des leucémies aiguës lymphoblastiques chez l'enfant et le jeune adulte, et de l'axicabtagene ciloleucel dans les lymphomes diffus à grandes cellules $B[3,4]$. Dans les tumeurs solides, les effets bénéfiques des CAR-T cells sont limités en raison d'une hétérogénéité antigénique tumorale rendant difficile la sélection d'une cible anti-tumorale pertinente, de la présence de LT du patient dysfonctionnels et surtout d'un microenvironnement tumoral (MET) défavorable au déclenchement d'une réponse anti-tumorale efficace, en empêchant la migration et l'infiltration de cellules immunocompétentes au sein de la tumeur et en favorisant en parallèle le recrutement de cellules immunosuppressives $[3,4]$. Autant d'obstacles qu'il faudra franchir afin de rendre ces CAR-T cells fonctionnelles dans les cancers solides, notamment par l'amélioration du ciblage des cellules, la définition d'une structure CAR adaptée et d'une cible antigénique adéquate [4]. Dans cette optique, la technologie CAR-T NKG2D ciblant des ligands de NKG2D, des Ag surexprimés par une grande variété de tumeurs, a été développée (Tableau 1) [5]. Des études précliniques ont démontré l'efficacité de ces cellules modifiées à détruire les cellules tumorales issues de différents types de cancers [4]. Cette cytotoxicité est associée à une production de cytokines pro-inflammatoires, telle que I'IFN(interféron) $-\gamma$ et I'IL(interleukine)-13 par les LT cytotoxiques et les cellules NK (natural killer), dans le MET [5].

\section{NKG2D/NKG2DL dans}

la surveillance immunologique des tumeurs solides

NKG2D est un récepteur activateur, exprimé par les cellules cytotoxiques de l'immunité innée et adaptative telles que les cellules NK, NKT, les LT CD8 ${ }^{+}$et les $\mathrm{LT} \gamma \delta$. Parmi ses ligands, on trouve, chez l'homme, les protéines ULBP (UL16-binding proteins) et les molécules MIC (MHC class-I-related molecule) A et $B$ [6]. Généralement, ces ligands sont absents ou très peu exprimés à la surface des cellules normales. En cas de stress cellulaire, d'inflammation ou de transformation tumorale, on observe une surexpression de ces ligands conduisant à l'activation de cellules effectrices NK et de certaines sous-populations de LT CD8 ${ }^{+}$via NKG2D [7]. Cependant, les tumeurs mettent en place un mécanisme d'échappement au système immunitaire, en clivant et en libérant les NKG2DL. En effet, il a été observé une augmentation de la concentration des NKG2DL dans le sérum de patients atteints de diverses tumeurs. Ces ligands circulants se lient aux récepteurs présents à la surface des cellules $\mathrm{NK}$ et $\mathrm{CD}^{+}$, limitant ainsi leur effet cytotoxique direct sur la cellule tumorale [7].

\begin{tabular}{|c|c|}
\hline Tumeurs solides & NKG2DL \\
\hline Gliomes primaires & 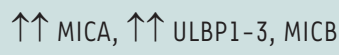 \\
\hline Carcinome ovarien & 个个 ULBP2, MICA, ULBPI, 3 \\
\hline $\begin{array}{l}\text { Les tumeurs primaires de carcinomes (poumon, sein, rein, } \\
\text { foie, prostate, ovaire et côlon), mélanome }\end{array}$ & $\uparrow \uparrow M I C A$ \\
\hline
\end{tabular}

Tableau I. Profil d'expression des ligands de NKGD2 (NKG2DL) sur certaines tumeurs solides [7, 8]. 


\section{NKG2D/NKG2DL dans}

le carcinome hépatocellulaire :

une cible anti-tumorale

Le carcinome hépatocellulaire ( $\mathrm{CHC}$ ) est un cancer primitif du foie très agressif, qui se développe dans 75 à $80 \%$ des cas sur une maladie chronique comme la cirrhose [9]. II correspond à la deuxième cause de mortalité liée au cancer dans le monde. Dans les premiers stades de la maladie, la chirurgie a un effet bénéfique pour les patients, mais avec une médiane de survie aux environs de 60 mois [10]. Aux stades plus avancés, la FDA a approuvé des inhibiteurs de tyrosine kinases (ITK) dont le sorafénib en première ligne, le régorafénib ou le cabozantinib en deuxième ligne. D'autres thérapies sont en cours d'approbation et d'évaluation dans des essais cliniques. La stratégie de l'utilisation des CAR-T cells, pour laquelle il est nécessaire d'identifier des biomarqueurs can- didats pour une prise en charge efficace du CHC, demeure peu explorée. L'analyse comparative de profils transcriptomiques d'échantillons de foie cancéreux de 32 patients et de tissus normaux, provenant de la base de données The Cancer Genome Atlas, a révélé une surexpression du MICA/B et ULBP 1, 2, 4 et 5 (NKG2DL) dans les échantillons de foie cancéreux. La surexpression du MICA a été observée chez 23 patients ( $72 \%$ ) et celle de l'ULBP2 chez 31 patients (97\%), avec absence de ces marqueurs dans les tissus de patients cirrhotiques non cancéreux [10]. Jusqu'à présent, aucun des Ag cibles associés aux tumeurs hépatiques évalués dans le cadre de thérapie à base de CAR-T cells n'a permis d'obtenir des réponses thérapeutiques [10]. Cependant, les observations décrites ci-dessus suggèrent que les NKGD2L pourraient être des candidats intéressants pour une immunothérapie à base de CAR-T cells NKG2D dans le CHC. $\varepsilon n$ se fondant sur ces observations, une étude récente de Sun et al., en 2019, a exploré l'efficacité d'une construction particulière de CAR-T cells ciblant les ligands de NKG2D dans un modèle préclinique de souris porteuses de xénogreffes de cellules tumorales de CHC [10]. Les LT ayant servi à la génération de CAR-T cells ont été isolés à partir de trois donneurs sains et de deux patients présentant un CHC. Dans cette étude, les cellules générées comportent le domaine extracellulaire du récepteur NKG2D pour les CAR-T cells NKG2D, ou un scFv anti-CD19 pour les CAR-T cells CD19 (témoins négatifs). Les deux constructions de CAR-T cells NKGD2D et CAR-T anti-CD19 portent les mêmes domaines intracellulaires composés du domaine de costimulation 4-1BB et du domaine de signalisation $\zeta$ CD3 (Figure 1).

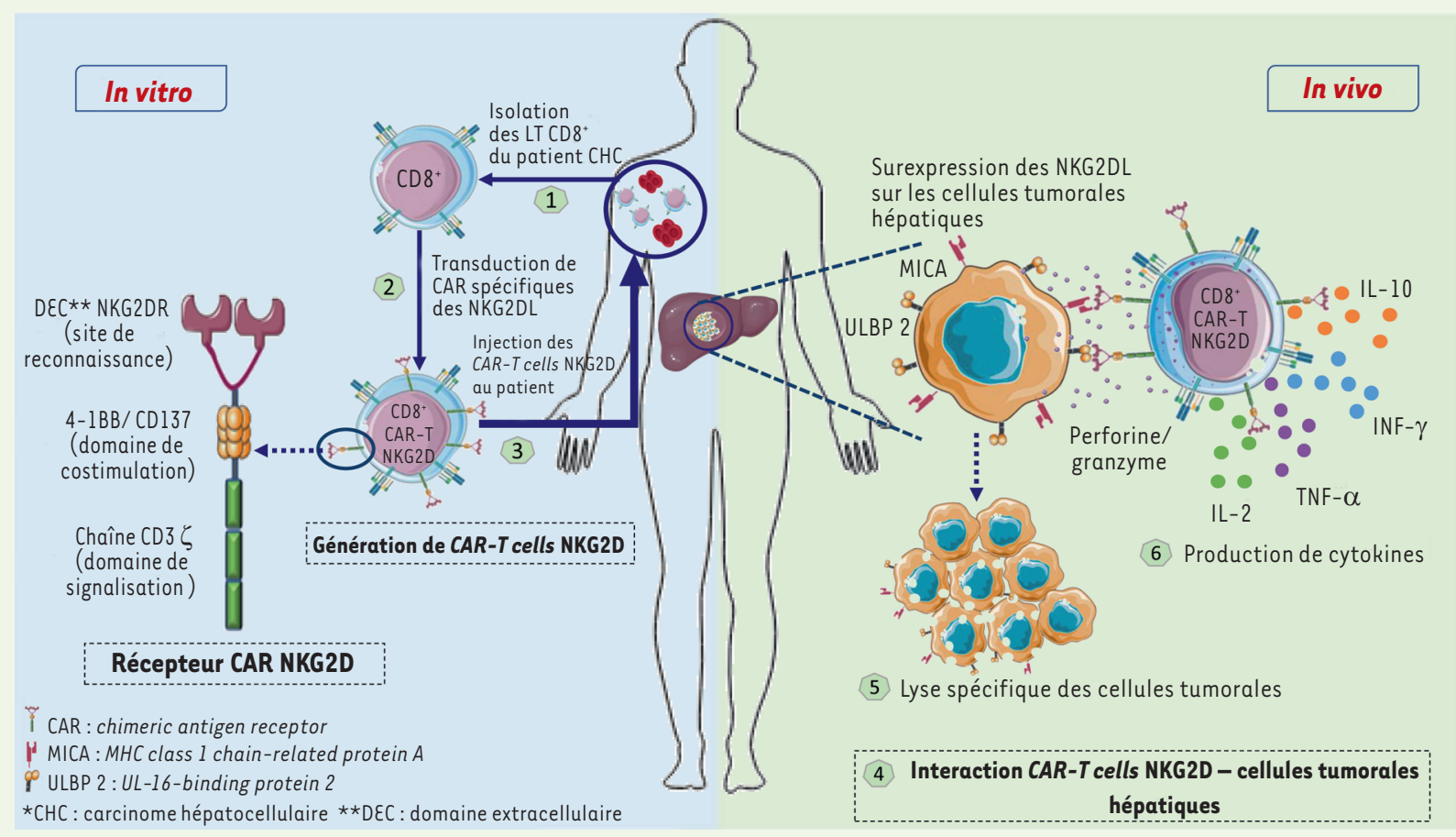

Figure 1. Génération, structure et interaction des CAR-T NKG2D avec les cellules tumorales hépatiques dans le traitement du carcinome hépatocellulaire (CHC). 1. Les $\mathrm{LT} C D 8^{+}$du patient CHC sont isolés à partir du sang périphérique. 2. Ces LT CD8 $8^{+}$sont modifiés génétiquement afin d'exprimer un récepteur chimérique qui reconnaît spécifiquement les ligands du récepteur NKG2D. Ce récepteur chimérique est composé d'un domaine extracellulaire (le récepteur NKG2D) et deux domaines intracellulaires (domaine de costimulation 4-1BB et domaine de signalisation $\zeta$ CD3) 3. Une fois générés, ces CAR-T cells NKG2D sont réinjectées au patient. 4. Les CAR-T cells NKG2D vont ensuite interagir spécifiquement avec les cellules tumorales hépatiques qui surexpriment NKG2DL au cours du carcinome hépatocellulaire. 5. L'action cytotoxique des CAR-T cells induit la lyse des cellules tumorales. 6. Les CAR-T cells vont produire également des cytokines telles que I'IL-2, le TNF- $\alpha$, I'IL-6, I'IL-10 et I'IFN- $\gamma$. 
Preuves expérimentales de l'efficacité des CAR-T cells NKG2D dans le carcinome hépatocellulaire

L'effet cytotoxique des différentes constructions de CAR $T$ cells générées a ensuite été analysé in vitro, en présence de trois lignées cellulaires de CHC : SMMC7721 et MHCC97H qui expriment fortement MICA et ULBP2, et Hep3b qui les exprime faiblement. Les résultats révèlent que pour un ratio effecteur: cible $(\varepsilon: T)$ de $8: 1$, les CAR-T cells CD19 n'induisent aucune lyse des cellules des lignées testées alors que les CAR-T cells NKG2D conduisent à la lyse de $100 \%$ des cellules SMMC-7721 et MHCC97H et de $30 \%$ des cellules Hep3b NKG2DL low. Une augmentation significative de la production des cytokines TNF- $\alpha$ (tumor necrosis factor alpha), IL-10, IL-2 et IFN- $\gamma$ est observée quand les cellules de la lignée SMMC-7721 sont mises en contact avec les CAR-T cells NKG2D.

Les résultats du test de cytotoxicité suggèrent que l'effet thérapeutique des CAR-T cells NKG2D serait fortement dépendant du niveau d'expression des NKG2DL. Dans le but de confirmer cette hypothèse, une surexpression ectopique de NKG2DL a été induite à la surface des cellules Hep3b. II a été observé une lyse de $60 \%$ contre $10 \%$ pour les cellules de la lignée sauvage au même ratio E:T. En parallèle, l'inhibition de l'expression des ligands, par transduction de séquences tronquées shMICA et ULBP2 dans les cellules des lignées SMMC-7721 et $\mathrm{MHCC} 97 \mathrm{H}$, diminue considérablement l'action cytotoxique des CAR-T cells NKG2D. Cependant, cette activité cytotoxique reste conservée dans le cas où un seul des deux ligands est absent. Les CAR-T cells NKG2D ciblent donc directement les cellules malignes qui surexpriment un ou plusieurs types de NKG2DL, couvrant ainsi l'hétérogénéité antigénique qui est observée dans les tumeurs, tout en protégeant les cellules saines qui expriment faiblement ces ligands. L'effet thérapeutique in vivo des CAR-T cells NKG2D a ensuite été évalué dans des modèles de souris portant une xénogreffe de cellules tumorales SMMC-7721. Les résultats ont montré que $100 \%$ des souris ayant reçu la thérapie CAR-T cells CD19 n'ont pas survécu plus de 27 jours, alors que $100 \%$ des souris traitées par les CAR-T cells NKG2D survivent au-delà de 53 jours, avec plus de la moitié des souris traitées en complète rémission et les autres présentant un faible résiduel de la masse tumorale. Parce qu'une interaction directe entre les CAR-T cells et les cellules tumorales est nécessaire à l'efficacité de la thérapie, la détection des CAR-T NKG2D par marquage immunohistochimique (IHC) du domaine CD3- $\zeta$, qu'elles expriment, réalisé sur les xénogreffes et les tissus sains, a révélé une localisation préférentielle des CAR-T cells NKG2D au sein de la tumeur.

Partant de l'hypothèse que les lymphocytes des patients CHC peuvent présenter des anomalies fonctionnelles, les auteurs ont testé dans un second temps les CAR-T cells NKG2D générés à partir des LT de patients $\mathrm{CHC}$, in vitro sur les lignées cellulaires et in vivo dans les souris portant les xénogreffes tumorales. Une activité cytotoxique et un effet thérapeutique, comparables à ceux obtenus avec des CAR-T cells des donneurs sains, ont été observés.

$\varepsilon \mathrm{n}$ conclusion, les CAR-T cells NKG2D ciblent les antigènes (NKG2DL) surexprimés presque exclusivement par les hépatocytes tumoraux au cours du CHC, permettant d'obtenir un effet cytotoxique spécifique, et par conséquent moins de toxicité et une protection des tissus sains, associée à une inhibition de la prolifération tumorale. Ces résultats décrivent dans leur globalité l'activité anti-tumorale de CAR-T cells NKG2D contre des cellules de lignées de cellules hépatocarcinomateuses. Cependant, l'utilisation élargie de ce type d'approche thérapeutique nécessite d'analyser plus amplement la toxicité des CAR-T cells NKGD2 et leurs effets secondaires. De plus, il s'avère nécessaire de prendre en considération les mécanismes d'échappement des tumeurs, qui ont la capacité de cliver et de libérer les NKG2DL dans le sang, qui en interagissant avec les récepteurs NKG2D des cellules cytotoxiques limitent leurs actions, ce qui constitue une limite à l'utilisation thérapeutique des CAR-T cells NKG2D [7]. Néanmoins, cette étude est intéressante car elle vient contourner certains obstacles qui s'opposaient à I'utilisation des CAR-T cells conventionnelles dans les tumeurs solides, et offre une preuve scientifique de l'efficacité de ce type d'approche dans le carcinome hépatocellulaire, ce qui ouvre un champ thérapeutique prometteur pour le traitement de ce type de cancer très agressif. $\diamond$ NKG2D CAR-T cells as an immunotherapy in hepatocellular carcinoma

\section{LIENS D'INTÉRÊT}

Les auteurs déclarent n'avoir aucun lien d'intérêt concernant les données publiées dans cet article.

\section{RÉFÉRENCES}

1. June CH, O'Connor RS, Kawalekar OU, et al. CAR-T cell immunotherapy for human cancer. Science 2018 ; $359: 1361-5$

2. Catros V. Les CAR-T cells, des cellules tueuses spécifiques d'antigènes tumoraux : de nouvelles générations pour le traitement des tumeurs solides. Med Sci (Paris) 2019 ; $35: 316-26$.

3. Schultz L, Mackall C. Driving CAR T cell translation forward. Sci Transl Med $2019 ; 11$.

4. Castellarin $\mathrm{M}$, Watanabe $\mathrm{K}$, June $\mathrm{CH}$, et al. Driving cars to the clinic for solid tumors. Gene Ther 2018; $25: 165-75$.

5. Sentman CL, Meehan KR. NKG2D CARs as cell therapy for cancer. Cancer J $2014 ; 20: 156-9$.

6. El-Gazzar A, Groh V, Spies T. Immunobiology and conflicting roles of the human NKG2D lymphocyte receptor and its ligands in cancer. J Immunol 2013 ; 191: 1509-15.

7. Spear P, Wu MR, Sentman ML, et al. NKG2D ligands as therapeutic targets. Cancer Immun 2013; $13: 8$.

8. Liu H, Wang S, Xin J, et al. Role of NKG2D and its ligands in cancer immunotherapy. Am J Cancer Res $2019 ; 9$ : 2064-78.

9. Blanc JF, Barbare JC, Baumann AS, et al. Carcinome hepatocellulaire. Thésaurus National de Cancérologie Digestive 2019.

10. Sun B, Yang D, Dai H, et al. NKG2D-BBz CAR-T eliminates hepatocellular cancer. Cancer Immunol Res $2019 ; 7: 1-11$.
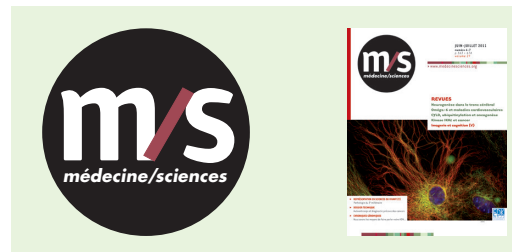

Abonnez-vous

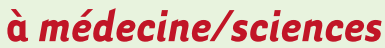

Bulletin d'abonnement page 678 dans ce numéro de $\mathrm{m} / \mathrm{s}$ 